\title{
When transfer-messenger RNA scars reveal its ancient origins
}

\section{Charlotte Guyomar and Reynald Gillet}

Univ. Rennes, CNRS, Institut de Génétique et Développement de Rennes (IGDR) UMR6290, 35000 Rennes, France.

Address for correspondence: Reynald Gillet, Université de Rennes 1, Institut de Génétique et Développement de Rennes, Bât. 4 - Campus de Villejean, 35000 Rennes, France. reynald.gillet@univ-rennes1.fr

Key words: alanine; genetic code; RNA world; tmRNA; trans-translation

Short title: tmRNA scars reveal its ancient origins

\begin{abstract}
In bacteria, trans-translation is the primary quality control mechanism for rescuing ribosomes arrested during translation. This key process is universally conserved and plays a crucial role in the viability and virulence of all bacteria. It is performed by transfermessenger RNA (tmRNA) and its protein partner small protein B (SmpB). Here we show that tmRNA is a key molecule that could have given birth to modern protein synthesis. The traces of an ancient RNA world persist in the structure of modern tmRNA, suggesting its old origins. Therefore, since it has both tRNA and mRNA functions, tmRNA could be the missing link that allowed modern genetic code to be read by the ribosome.
\end{abstract}




\section{RNA world and protein synthesis}

The RNA world hypothesis is based on the ability of RNA to be a ribozyme, an entity which simultaneously acts as a genetic polymer and as a catalyst ${ }^{1}$. This (partially) solves the chicken-and-egg dilemma of "which came first, DNA or proteins?," since RNA can be both the "chicken" and the "egg" (Fig. 1). This would imply that before life appeared on earth, abiotic reactions gradually generated the components for biogenic processes. A collection of small molecules then progressively interacted with each other through a process of evolution that we can call "Molecular Darwinism" ${ }^{2}$. The abiotic synthesis of monomers, oligomers, and supramolecular systems was made possible by ensembles of molecules, the prebiotic pools ${ }^{3}$. The first RNA molecules could have been formed directly from these primitive syntheses, through a prebiotic evolution leading to the RNA world ${ }^{4}$. Even though a time machine allowing a precise description of the RNA world does not exist, we can look for the molecular traces of these ancient times in modern life, just as paleontologists do when they study fossils to determine the evolution of organisms. And indeed, RNA still plays many key roles in most of the current cellular processes ${ }^{5}$. This theory rings especially true for the process of protein synthesis, which is carried out by the ribosome, a complex modern ribonucleoprotein machine. In fact, it is now clear that ribosomal RNA forms the foundation for the reactor core which supports the three central roles of the ribosome, i.e. aminoacyltRNA selection, peptide bond formation catalysis, and mRNA and tRNA coupled translocation ${ }^{6}$. The conserved nucleotides G530, A1492, and A1493 in the decoding site (using E. coli numbering) permit the correct geometry of codon-anticodon interactions to take place during tRNA selection, with the conserved nucleotides in the large subunit's peptidyl center allowing for peptide bond formation catalysis. Finally, on a larger-scale level, coordinated movements between the RNA from both ribosomal subunits enables the dynamics of the coupled translocation of mRNA and tRNA. The peptidyltransferase center is the most important feature of protein synthesis. It is a universal symmetrical pocket made up exclusively of RNA and formed by the dimerization of two "stem-elbow-stem" RNA motifs

(Fig. 2). This pocket is certainly a relic, and may have occurred spontaneously by gene duplication or gene fusion ${ }^{7}$. An ancient proto-ribosome is supposed to have driven the catalytic reactions that were necessary to polymerize the first amino acid or amino acid-like molecules. Such a proto-ribosome definitely evolved in parallel to the genetic code, giving birth to the complex process that we know today 8 . 


\section{Back to the future: which came first, tRNA or mRNA?}

In the search for the usual suspects that could have permitted the rapid and parallel evolutions of the genetic code and ribosome-based protein synthesis, transfer RNA (tRNA) is often cited as the perfect key intermediate. These molecules bring together two different genetic codes and link DNA to peptides. Indeed, each tRNA has an anticodon, a triplet of nucleotides which correspond to an mRNA codon, but also has specific signatures governing its aminoacylation by the corresponding aminoacyl-tRNA synthetase (aaRS) ${ }^{9}$. However, and as recently mentioned by Massimo Di Giulio ${ }^{10}$, the genetic code certainly resulted from successions of interactions between peptidated-RNA and pre-mRNAs. Here, mRNAs play a central role in the sense that they started coding and therefore directed the interactions between peptidated-RNAs. In this scenario, peptidyl-tRNA would have arrived later on, a result of peptidated-RNA evolution. Returning to the paleontological approach, relics from these molecules should still be present in our modern life, where mRNAs specify the codes for protein amino acid sequences and are read by tRNA within the framework of the ribosomal machinery. Strikingly, if we focus our attention on the ribosome, it clearly carries all the traces expected for a molecular machine coming from an ancient world, as detailed above. However, when looking at tRNA and mRNA, it is quite difficult to elaborate a scenario connecting these two entities in an RNA world. But why do we have to choose between tRNA and mRNA to explain the emergence of peptidated-RNA? Indeed, in modern life, the fantastic hybrid molecule of transfer-messenger RNA (tmRNA) has the properties of both of its partners in a single entity. It might therefore be the key molecule that gave birth to modern protein synthesis, evolving in parallel with and then together with a proto-ribosome also made of RNA.

\section{Transfer-messenger modern structure and functions}

tmRNA is the principal actor of trans-translation, the main pathway rescuing stalled ribosomes in bacteria. The process occurs very frequently and is highly efficient, accounting for as much as $2-4 \%$ of translation reactions in $E$. coli $i^{11-12}$. Because trans-translation is a universally conserved system ${ }^{13}$, and because mutants without it display deficient phenotypes or cell death ${ }^{14}$, this unique pathway obviously gives bacteria a substantial selective advantage. 
tmRNA is an uncommon hybrid RNA with a dual function, since it acts simultaneously as a transfer RNA and as a messenger RNA ${ }^{15}$. The $3^{\prime}$ and $5^{\prime}$ termini of tmRNA form the tRNA-like domain (TLD) while the internal open reading frame forms an mRNA-like domain (MLD). A large portion of the molecule then folds into RNA pseudoknots. The tRNA-like domain (TLD) structure is similar to that of a canonical tRNA, with high conservation of the T stem-loop and the acceptor arm that allows specific aminoacylation by alanyl-tRNA synthetase (AlaRS, see below) (Fig. 3A). On the other hand, and contrary to canonical tRNA, the D stem-loop is replaced by a simple D-loop, and the anticodon loop is replaced by a helix extended by a large loop made of four pseudoknots (PK1 to PK4), two helices (H2 and H5), and the MLD. The MLD is always located between PK1 and PK2, and encodes for a specific amino acid sequence varying in length from 10 to 35 amino acid residues (ANDENYALAA in E. coli) that is recognized by proteases and therefore triggers the destruction of incomplete polypeptides. The first codon GCA (alanine) is highly conserved ${ }^{16}$, as is the upstream region necessary for the perfect positioning of the MLD. Interestingly, while this tmRNA structure is highly conserved in bacterial phyla, an uncommon form of "two-piece tmRNA" that breaks the mRNA domain loop has been observed in alphaproteobacteria and cyanobacteria, and rarely in some betaproteobacteria. This rearrangement results from independent gene permutation events that certainly benefit the functioning of $\operatorname{tmRNA}{ }^{17-19}$.

How does the highly structured tmRNA deliver stalled ribosomes? During trans-translation, tmRNA acts in concert with its partner, small protein B (SmpB). SmpB binds to the D-loop of the $\operatorname{TLD}^{20-21}$ to mimic a canonical tRNA anticodon loop structure. As with canonical translation, the tmRNA/SmpB complex is brought to the ribosome by the elongation factor Tu bound to GTP (EF-Tu•GTP). The stalled ribosome's vacant A-site is recognized by SmpB, which inserts and folds its C-terminal tail in the empty ribosomal mRNA path downstream of the truncated $m \mathrm{RNA}^{22}$. After accommodation, the CCA-3' extremity of the TLD enters the peptidyl transferase center, allowing the stuck nascent peptide to be transferred from the Psite tRNA to the A-site's Ala-tmRNA. The defective non-stop mRNA is then degraded by RNase $R$ and translation resumes on the tmRNA MLD, continuing in the usual manner until it arrives at the internal termination codon. At the end of the process, the stalled ribosomes are recycled, while the incomplete peptides are tagged and consequently destroyed by specific proteases (Fig. 4). 


\section{Transfer-Messenger RNA scars}

\section{Within the tRNA-likedomain (TLD)}

The old G3:U70 base pair signature

The tRNA-like domain of tmRNA is deeply linked to alanine-specific tRNA (Fig. 3A). Indeed, tmRNA is always aminoacylated by alanyl-tRNA synthetase (AlaRS), a class II tRNA synthetase that also catalyzes the esterification of alanine to tRNA ${ }^{\text {Ala }}$. Throughout the three domains of life, AlaRS recognizes G3:U70 base pairs in the acceptor stem of tRNA ${ }^{\text {Ala }}$ as the major identity determinants for subsequent aminoacylation ${ }^{23}$. This is unusual, since the most obvious way for an aaRS to recognize a specific tRNA would be through direct recognition of the tRNA anticodon ${ }^{24}$. However, the anticodon is not actually used as the principal determinant for AlaRS aminoacylation, and no physical contacts occur between the enzyme and the tRNA anticodon. Consequently, small RNA helices that contain a G3:U70 base pair are robust substrates for alanylation. This type of recognition is perfect for tmRNA, which do not have an anticodon. Furthermore, this reflects the lifespan of tmRNA since this operational RNA code, also called the "second genetic code" ${ }^{25}$, is likely to have predated or even be the progenitor of the current genetic code ${ }^{26}$.

\section{The intron-like scenario}

The absence of an anticodon stem-loop next to the tmRNA TLD is necessary for its functioning (see above). However, this also raises questions about the origins of tmRNA as compared to tRNA. Is the large and complicated tmRNA the ancestor of the modern and simpler tRNAs after a process of simplification and specialization? Or, on the contrary, is it the result of the evolution of an ancient $\mathrm{TRNA}^{\text {Ala }}$ that became more complex after the addition of a long fragment? To try to answer to this question, it is again interesting to look at the traces, but this time in modern tRNA. Surprisingly, this situation is not so rare: in the three domains of life, several canonical tRNA molecules have large introns within their anticodon stem-loops ${ }^{27-28}$. In these tRNAs, the active anticodon loop is formed only after splicing of the non-coding sequences. Strikingly, in bacteria, these introns belong to selfsplicing group I introns, known to be very old since they exhibit self-catalytic activities characteristic of the RNA world. Therefore, in an "introns-early" scenario, all ancient tRNA genes would have an intermediate and self-removable block like this in their anticodon 
loops $^{29}$. These blocks progressively disappeared throughout evolution, and now only traces remain. In such a scenario, the analogy with modern tmRNA is obvious, and these could be the result of an ancient molecule of that type that lost its ability to act as a cleavable intron instead of losing its intermediate block. Indeed, when looking at the sizes, sequences, and positions of the introns in the secondary structure of modern tRNA, the introns correspond to the large RNA structure which replaces the anticodon stem-loop in the tmRNA TLD ${ }^{30}$. Therefore, an ancient "proto-tmRNA" could be the common ancestor of both present-day tmRNA and tRNA molecules.

\section{Within the internal open reading frame}

The alanine codons

During the initiation step of canonical translation, the ribosome recruits an mRNA and selects the start codon of the open reading frame (ORF). In bacteria, this corresponds to the

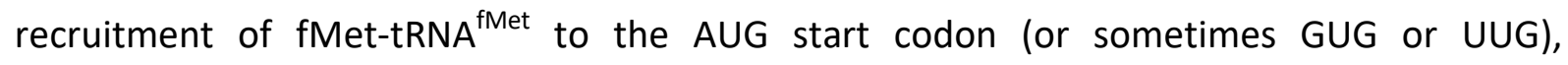
promoted by initiation factors IF1, IF2, and IF3 (for a recent review, see Ref. 8). Strikingly, tmRNA uses neither a methionine codon nor initiation factors to correctly place the reading frame and resume translation. Instead, translation restarts on the internal ORF whose resume codon is correctly positioned in the decoding site after specific interactions of tmRNA with its protein partner SmpB. Perhaps most surprising is the fact that this resume codon is never a methionine codon, but in most cases is an alanine or even a glycine codon. Codes for alanine and glycine, the two simplest amino acids, were presumably the first to be integrated in the genetic code ${ }^{31}$. In a recent work, Grosjean and Westhof $f^{32}$ proposed a new and very clever representation of the genetic code table, suggesting that the modern genetic code could have emerged from an ancient GC-rich one. In this scheme, Ala codons belong (along with Arg, Gly, and Pro) to the small group of codons that only have strong $\mathrm{C}=\mathrm{G}$ or $\mathrm{G}=\mathrm{C}$ base pairings at the first and second base pairs of the codon/anticodon helix. The earliest proto-biosynthetic system would thus have originated from RNA:RNA duplexes, with the most stable complementary GC-rich triplets coding for small polypeptides composed of alternating hydrophobic alanines and hydrophilic glycines, the very first amino acids encoded $^{32}$. Accordingly, when comparing tmRNA resume codon sequences among bacterial species, we see that the more primitive the bacteria, the more ancestral GNC (where $N$ is $\mathrm{A}$, $\mathrm{U}, \mathrm{C}$, or $\mathrm{G}$ ) codons are used ${ }^{30}$. Three other conserved and indispensable Ala codons follow 
the resume codon at the $3^{\prime}$-end of the internal ORF whose consensus sequence is "AN-----ALAA," and this must reflect other traces of the older proto-tmRNA. These three alanines are crucial for trans-translation, as their specific recognition by proteases permits the degradation of the incomplete peptide after tagging (see above).

\section{Stopping within a stem-loop}

Interestingly, the tmRNA termination codon is always embedded within a conserved $\mathrm{H} 5$ hairpin stem-loop. The primitive punctuation of this termination signal in the RNA world consisted of such multidimensional structures allowing for recognition by DNA and RNA polymerases $^{33}$. These punctuation marks were then certainly hijacked for translation. The tmRNA H5 helix, located at the end of the internal open reading frame, could thus be a remnant of an ancient termination signal now including a modern stop codon.

\section{Within the internal pseudoknot-loop}

tmRNA is a highly structured molecule encompassing the two functional modules TLD and MLD, but also featuring four pseudoknot structures (PK1 to PK4) that form a large and conserved closed loop containing the MLD. Pseudoknots result from base pairings between nucleotides that are within a loop to complementary nucleotides outside of the loop ${ }^{34}$. PK1 is located upstream from the MLD and is considered to be the only pseudoknot essential for tmRNA function. However, its role is purely structural, as it can be replaced with a variety of hairpin structures ${ }^{35}$. In a similar way, without serious effects on tmRNA functions, all of the other pseudoknots can be replaced by single-stranded RNA (at least in E. coli) or can even be interchanged $^{36}$. We can therefore consider that their conservations are the traces of ancient evolutionary processes that today ensure tmRNA stability. Indeed, pseudoknots are elements that could have emerged from abundant stem-loop and hairpin modules through kissing-loop interactions (loop-loop contacts) in the RNA world ${ }^{37}$.

\section{Conclusion}

In the search for the missing link(s) between modern genetic code and the process of translation, like paleontologists we looked at modern molecules for the scars of an ancient RNA world. As a proof of their links to the ancient times, such molecules would need to be catalytic, ubiquitous, and/or central to some aspect of metabolism ${ }^{38-39}$. Among the 
numerous modern RNA molecules that fulfill these criteria, tmRNA stands out. It is a powerful and central component, and able to play the two main roles (tRNA and mRNA) indispensable to the emergence of ribosome-driven protein synthesis. In fact, all along the structure of tmRNA we can find relics from old times, including the tRNA-like domain, the mRNA like-domain, and even the instrumental big loop formed by RNA pseudoknots. During primordial times, short RNA and ribonucleotides must have played the first catalytic and simultaneously structural roles. Considering the great versatility of ribonucleotides, short RNAs would have tended to fold or to pair with each other, forming minihelical (stem-loop) structures. Homo- and heterodimerization between two minihelices would have led on one hand to the emergence of the first proto-tmRNA, and to the first peptidyl-transferase center (PTC) on the other (Fig. 5). At that stage, proto-tmRNA may have acted as the first peptidated-mRNA ${ }^{10}, 40$ drivingprimitive amino acid transfer independently of protoribosomes. Indeed, we can assume that these proto-tmRNAs could carry not only alanine but also various other early amino acids, depending on their acceptor branches. The large loop (closed or possibly broken as in modern two-piece tmRNAs) prolonging the aminoacylated parts of proto-tmRNA would then act as a strand of primitive codons, interacting with the nucleotides from the loops in other proto-tmRNAs. This would have been simply done by creating antiparallel duplexes, as is the case for modern anticodon-codon interactions ${ }^{41}$. Later on, binding of prebiotic oligopeptides with basic amino acids would stabilize these RNA, leading to the first ribonucleoprotein complexes, which would then coevolve into more complex and efficient ribozymes ${ }^{1}$. However, since co-evolution of these two entities (prototmRNA and proto-ribosomes) seems indispensable for the emergence of an early genetic code, it would need a final convergence allowing the genetic code to be processed by the complex ribosomal machinery. This convergence implies that the two functions of tmRNA, tRNA and mRNA, would need to split at that point to permit an accurate regulation of translation at the ribosomal level. However, despite the appearance of tRNA and mRNA, it is interesting to note that tmRNA was maintained across the ages, presumably to cope with the highly frequent translational errors during the emergence of the process of translation.

Today, all bacteria (but no other life types) have tmRNA, suggesting that this function was lost in the eukaryotic and archaeal lineages with the appearance of ubiquitin ${ }^{30}$. It is noteworthy that the scars of an ancient RNA world still persist in all modern tmRNAs, 
suggesting not only their very old origins, but also the necessity of these signatures for the functioning of modern tmRNA.

\section{Acknowledgments}

This work was supported by the Direction Générale de l'Armement (DGA) and the Agence Nationale pour la Recherche (No. ANR-14-ASTR-0001). Charlotte Guyomar was supported by the DGA and the Université de Rennes 1 (PhD financial support). The authors are particularly grateful to Emmanuel Giudice for his invaluable advice and the figures, and to Juliana Berland for insightful comments on the manuscript.

\section{Conflicts of interest}

The authors declare no conflicts of interest.

\section{Figures:}

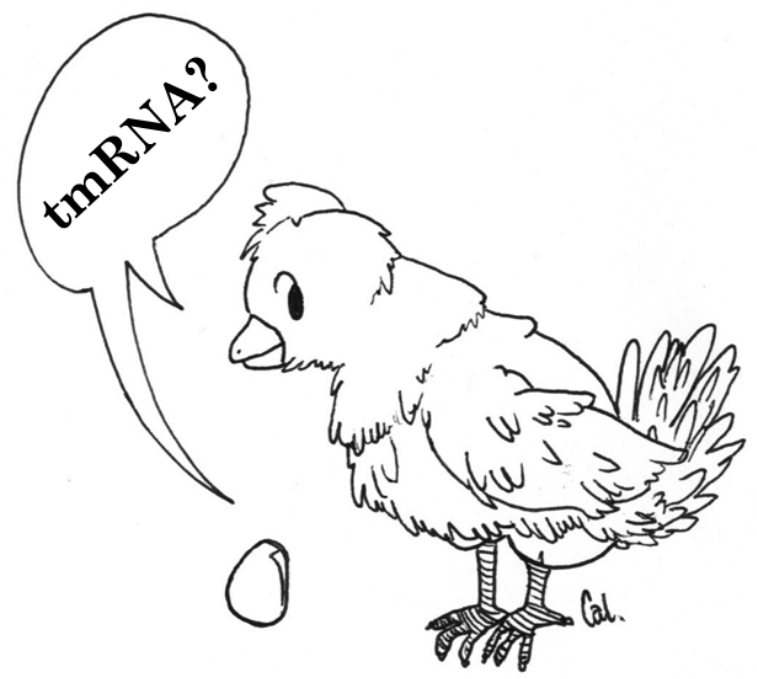

Figure 1. The chicken-and-egg dilemma of "which came first?" 


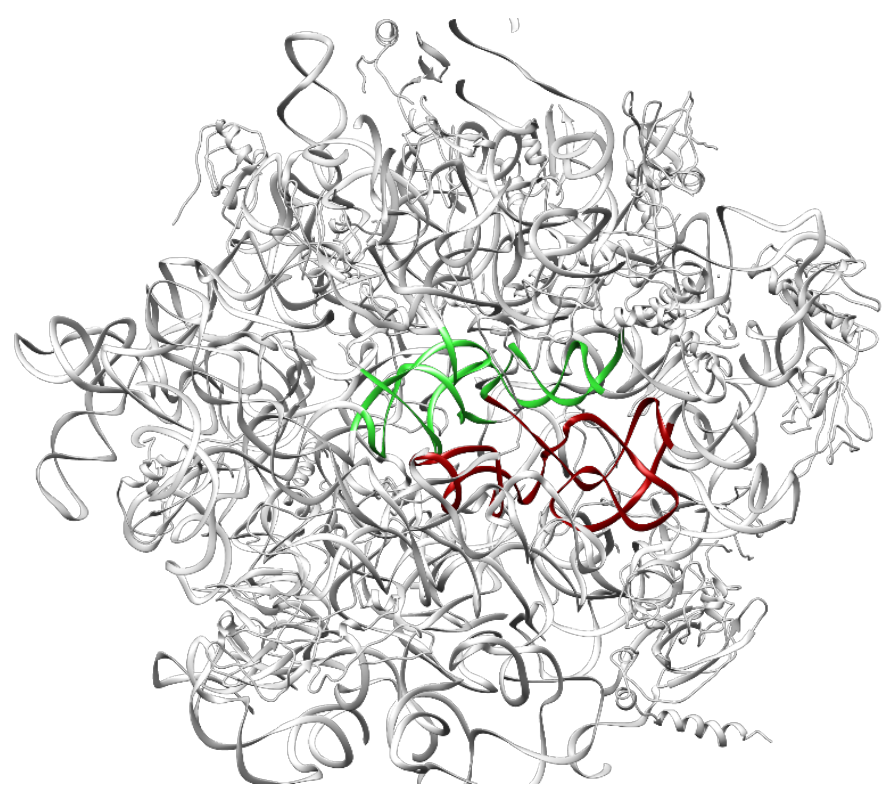

Figure 2. Ribosomal symmetry. The ribosome is light gray (Protein Data Bank code 4V6F). The green and dark red helices indicate the double helix that forms the symmetrical region of the PTC cavity (adapted from Belousoff et al., 2010).

A

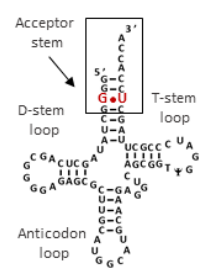

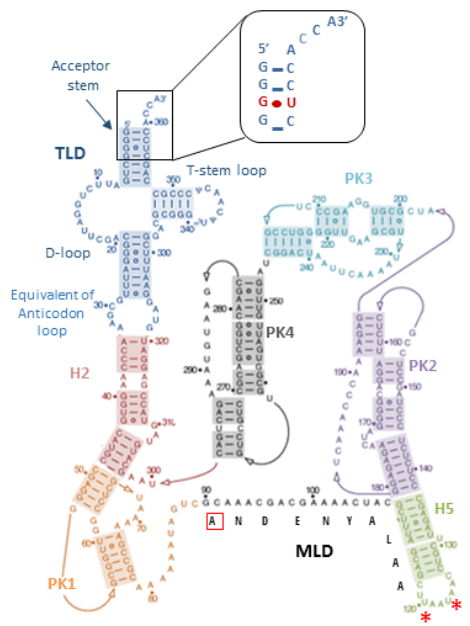

B

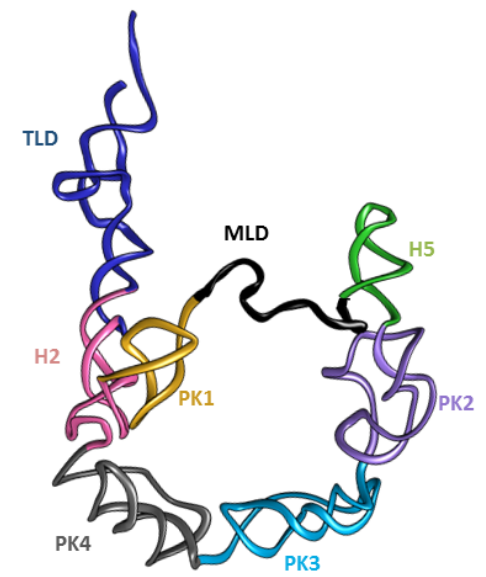

Figure 3: Structure of tmRNA. (A) Secondary structure of tmRNA (right) compared to the structure of alanine-specific tRNA (left). Indicated are the two functional domains TLD (dark blue) and MLD (black), the $\mathrm{H} 2$ (pink) and $\mathrm{H} 5$ (green) helices, and the pseudoknots PK1 (orange), PK2 (purple), PK3 (light blue), and PK4 (gray). The two acceptor stems of tmRNA and tRNAAla are framed in black and a close up on the third wooble base pairing (in red) is shown. It allows for aminoacylation by AlaRS. The red stars indicate the two successive stop codons present in E. coli tmRNA (B) 3D structure of tmRNA, using the same color code. 


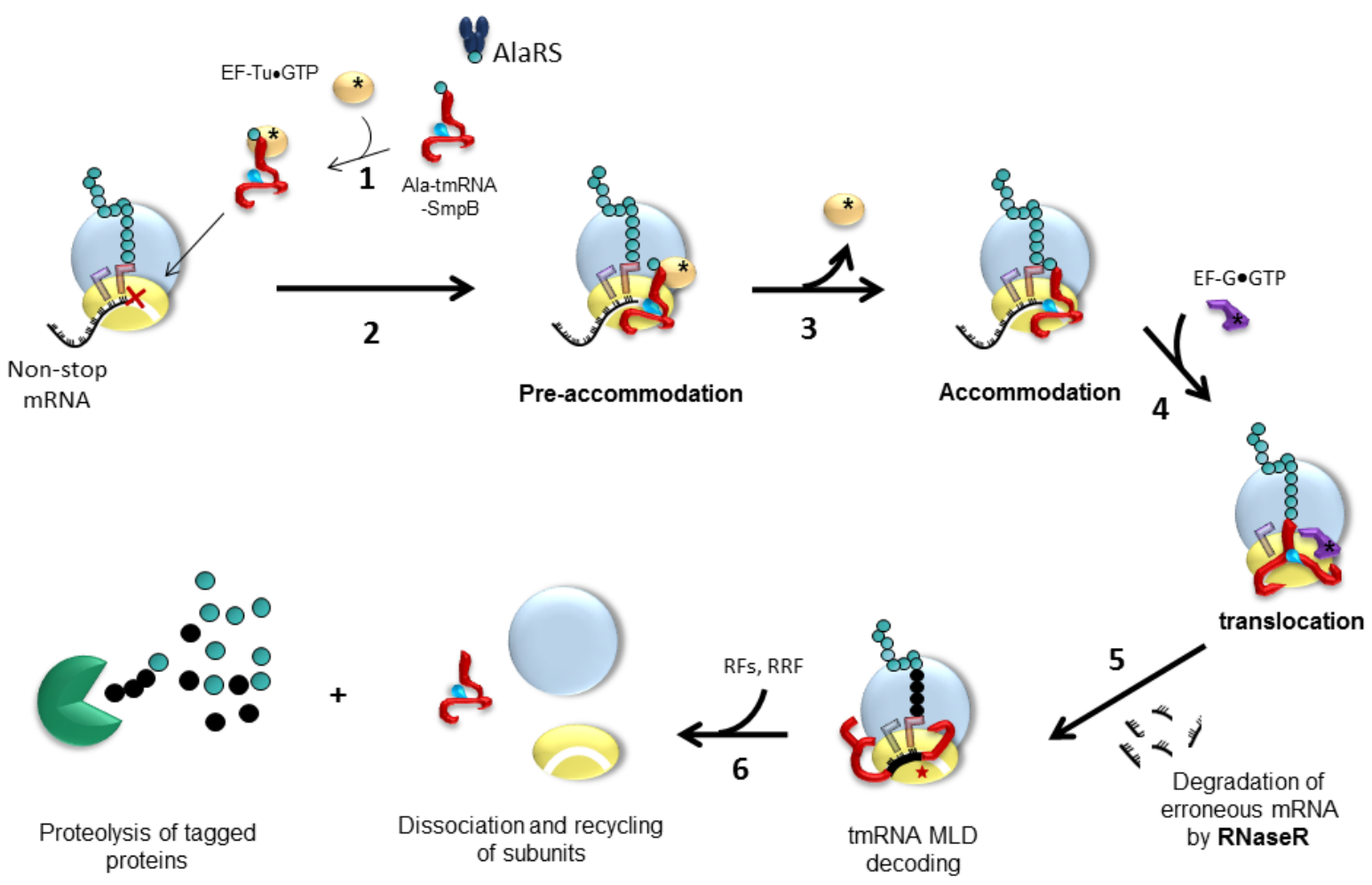

Figure 4: The trans-translation mechanism. (1) After aminoacylation of tmRNA by alanyltRNA synthetase (AlaRS), the quaternary complex ala-tmRNA-SmpB-EF-Tu•GTP is formed. (2) Pre-accommodation step: The quaternary complex recognizes the vacant A-site thanks to the C-terminal tail of SmpB. (3) Accommodation step: EF-Tu is released after the hydrolysis of GTP into GDP. Ala-tmRNA-SmpB accommodates into the A-Site and the peptidyl transfer occurs on the TLD. (4) Translocation step: After peptidyl transfer of the nascent peptide from the P-Site tRNA to the ala-tmRNA, EF-G・GTP binds to the ribosome. TLD-SmpB and tRNA are translocated to the $\mathrm{P}$ - and E-sites, respectively. tmRNA's resume codon is then perfectly positioned in the A-site for decoding (5). In this step, the defective non-stop mRNA is degraded by RNase R. (6) Elongation and termination: canonical translation resumes on the MLD until the ribosome reaches the termination codon, within helix $\mathrm{H} 5$. The tagged peptide is released, and the two ribosomal subunits are dissociated to be recycled. The tagged peptide is then recognized by proteases and degraded. 


\section{Ribonucleotides}

\section{and small RNAs}

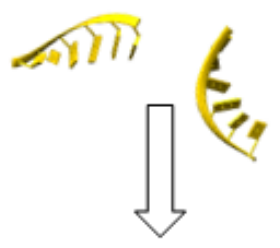

\section{Minihelices}
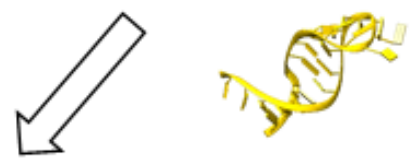

\section{Proto-tmRNAs}

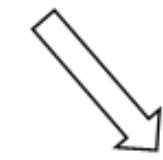

\section{Proto-ribosome}

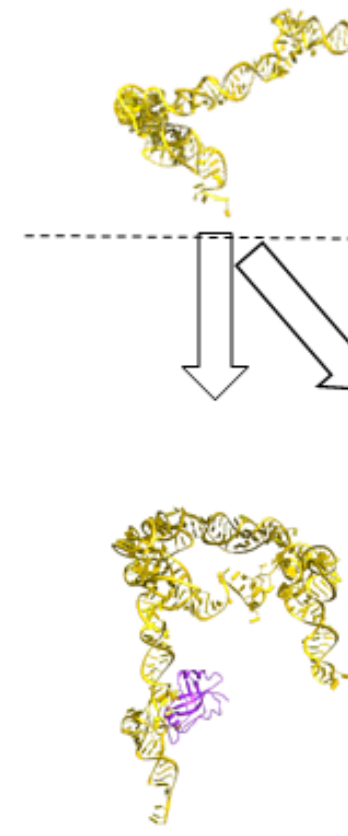

Primitive protein synthesis

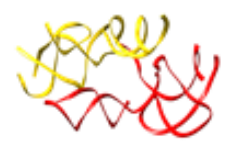

\section{Contemporary protein synthesis}

tmRNA

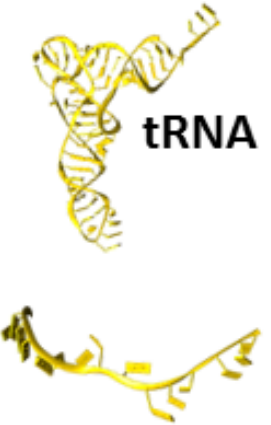

mRNA

Ribosome

Figure 5. tmRNA as a key player when switching from ancestral to modern protein synthesis 


\section{References}

1. Vázquez-Salazar, A. \& A. Lazcano. 2018. Early Life: Embracing the RNA World. Curr. Biol. 28: R220R222.

2. Saladino, R., J.E. Šponer, J. Šponer, et al. 2018. Chemomimesis and Molecular Darwinism in Action: From Abiotic Generation of Nucleobases to Nucleosides and RNA. Life (Basel) 8: 24.

3. Lazcano, A. 2018. Prebiotic Evolution and Self-Assembly of Nucleic Acids. ACS Nano. 12: 96439647.

4. Robertson, M.P. \& G.F. Joyce. 2012. The Origins of the RNA World. Cold Spring Harb. Perspect. Biol. 4: a003608.

5. Doudna, J.A. \& T.R. Cech. 2002. The chemical repertoire of natural ribozymes. Nature 418: 222228.

6. Noller, H.F. 2017. The parable of the caveman and the Ferrari: protein synthesis and the RNA world. Philos. Trans. R. Soc. Lond. B. Biol. Sci. 372: 20160187.

7. Belousoff, M.J., C. Davidovich, E. Zimmerman, et al. 2010. Ancient machinery embedded in the contemporary ribosome. Biochem. Soc. Trans. 38: 422-427.

8. Rodnina, M.V. 2018. Translation in Prokaryotes. Cold Spring Harb. Perspect. Biol. 10: a032664.

9. Shepherd, J. \& M. Ibba. 2015. Bacterial transfer RNAs. FEMS Microbiol. Rev. 39: 280-300.

10. Di Giulio, M. 2015. A Model for the Origin of the First mRNAs. J. Mol. Evol. 2015 81: 10-17.

11. Moore, S.D. \& R.T. Sauer. 2005. Ribosome rescue: tmRNA tagging activity and capacity in Escherichia coli. Mol Microbiol. 58: 456-66.

12. Ito, K., Y. Chadani, K. Nakamori, K., et al. 2011. Nascentome analysis uncovers futile protein synthesis in Escherichia coli. PLoS One 6: e28413.

13. Whithey, J.H. \& D.I. Friedman. 2003. A salvage pathway for protein structures: tmRNA and transtranslation. Annu. Rev. Microbiol. 57: 101-23.

14. Keiler, K.C. \& H.A. Feaga. 2014. Resolving Nonstop Translation Complexes Is a Matter of Life or Death. J. Bacteriol. 196: 2123-2130.

15. Giudice, E., K. Macé \& R. Gillet. 2014. Trans-translation exposed: understanding the structures and functions of tmRNA-SmpB. Front. Microbiol. 5: 113.

16. Kapoor, S., L. Samhita \& U. Varshney. 2011. Functional significance of an evolutionarily conserved alanine (GCA) resume codon in tmRNA in Escherichia coli. J. Bacteriol. 193: 3569-3576.

17. Keiler, K.C., L. Shapiro \& K.P. Williams. 2000. tmRNAs that encode proteolysis-inducing tags are found in all known bacterial genomes: A two-piece tmRNA functions in Caulobacter. Proc Natl Acad Sci U S A. 97: 7778-7783. 
18. Gaudin, C., X. Zhou, K.P. Williams et al. 2002. Two-piece tmRNA in cyanobacteria and its structural analysis. Nucleic Acids Res. 30: 2018-2024.

19. Sharkady, S.M. \& K.P. Williams. 2004. A third lineage with two-piece tmRNA. Nucleic Acids Res. 32: 4531-4538.

20. Gutmann, S., P.W. Haebel, L. Metzinger, et al. 2003. Crystal structure of the transfer-RNA domain of transfer-messenger RNA in complex with SmpB. Nature 424: 699-703.

21. Bessho, Y., R. Shibata, S. Sekine et al. 2007. Structural basis for functional mimicry of longvariable-arm tRNA by transfer-messenger RNA. Proc Natl Acad Sci U S A. 104: 8293-8298.

22. Neubauer, C., R. Gillet, A.C. Kelley et al. 2012. Decoding in the absence of a codon by tmRNA and SmpB in the ribosome. Science 335: 1366-1369.

23. Chong, Y.E., M. Guo, X.L. Yang, et al. 2018. Distinct ways of G:U recognition by conserved tRNA binding motifs. Proc. Natl. Acad. Sci. U S A. 115: 7527-7532.

24. Ribas de Pouplana, L. \& P. Schimmel. 2001. Operational RNA code for amino acids in relation to genetic code in evolution. J. Biol. Chem. 276: 6881-6884.

25. de Duve, C. 1988. Transfer RNAs: the second genetic code. Nature 333: 117-118.

26. Musier-Forsyth, K. \& P. Schimmel. 1999. Atomic Determinants for Aminoacylation of RNA Minihelices and Relationship to Genetic Code. Acc. Chem. Res. 32: 368-375.

27. Abelson J., C.R. Trota \& H. Li. 1998. tRNA splicing. J. Biol. Chem. 273: 12685-12688.

28. Kanai, A. 2015. Disrupted tRNA Genes and tRNA Fragments: A Perspective on tRNA Gene Evolution. Life Basel Switz 5: 321-331.

29. Di Giulio, M. 1992. On the origin of the transfer RNA molecule. J. Theor. Biol. 159: 199-214.

30. Macé, K. \& R. Gillet. 2016. Origins of tmRNA: the missing link in the birth of protein synthesis? Nucleic Acids Res. 44: 8041-8051.

31. Trifonov, E.N. 2000. Consensus temporal order of amino acids and evolution of the triplet code. Gene 216: 139-151.

32. Grosjean, H. \& E. Westhof. 2016. An integrated, structure- and energy-based view of the genetic code. Nucleic Acids Res. 44: 8020-8040.

33. El Houmami, N. \& H. Seligmann. 2017. Evolution of Nucleotide Punctuation Marks: From Structural to Linear Signals. Front. Genet. 8: 36.

34. Pleij, C.W.A. 1994. RNA pseudoknots. Curr. Op. Struct. Biol. 4: 337-344.

35. Tanner, D.R., J.D. Dewey, M.R. Miller M.R., et al. 2006. Genetic analysis of the structure and function of transfer messenger RNA pseudoknot 1. J. Biol. Chem. 281: 10561-10566. 
36. Nameki, N., T. Tadaki T., H. Himeno, et al. 2000. Three of four pseudoknots in tmRNA are interchangeable and are substitutable with single-stranded RNAs. FEBS Lett. 470: 345-349.

37. Briones, C., M. Stich \& S.C. Manrubia. 2009. The dawn of the RNA World: Toward functional complexity through ligation of random RNA oligomers. RNA 15: 743-749.

38. Jeffares, D.C., A.M. Poole \& D. Penny. 1998. Relics from the RNA world. J. Mol. Evol. 46: 18-36.

39. Meli, M., B. Albert-Fournier \& M.C. Maurel. 2001. Recent findings in the modern RNA world. Int Microbiol. 4: 5-11.

40. Gillet, R. \& B. Felden. 2001. Transfer RNA(Ala) recognizes transfer-messenger RNA with specificity; a functional complex prior to entering the ribosome? EMBO J. 20: 2966-2976.

41. Lacey, J.C. \& Staves, M.P. 1990. Was there a universal tRNA before specialized tRNAs came into existence? Orig. Life Evol. Biosphere J. Int. Soc. Study Orig. Life 20: 303-308. 